\title{
Avian sex chromosomes: dosage compensation matters
}

\author{
Heather A. McQueen • Michael Clinton
}

(C) Springer Science + Business Media B.V. 2009

\begin{abstract}
In 2001 it was established that, contrary to our previous understanding, a mechanism exists that equalises the expression levels of $\mathrm{Z}$ chromosome genes found in male (ZZ) and female (ZW) birds (McQueen et al. 2001). More recent large scale studies have revealed that avian dosage compensation is not a chromosome-wide phenomenon and that the degree of dosage compensation can vary between genes (Itoh et al. 2007; Ellegren et al. 2007). Although, surprisingly, dosage compensation has recently been described as absent in birds (Mank and Ellegren 2009b), this interpretation is not supported by the accumulated evidence, which indicates that a significant proportion of $\mathrm{Z}$ chromosome genes show robust dosage compensation and that a particular cluster of such dosage compensated genes can be found on the short arm of the $\mathrm{Z}$ chromosome. The implications of this new picture of avian dosage compensation for avian sex determination are dis-
\end{abstract}

Responsible Editor: Edith Heard.

\footnotetext{
H. A. McQueen $(\bowtie)$

Institute of Cell Biology University of Edinburgh,

West Mains Rd,

Edinburgh EH9 3JR, UK

e-mail: h.mcqueen@ed.ac.uk

M. Clinton

Division of Developmental Biology,

Roslin Institute and R(D)SVS, Edinburgh University,

Midlothian EH25 9PS, UK

e-mail: michael.clinton@roslin.ed.ac.uk
}

cussed, along with a possible mechanism of avian dosage compensation.

Keywords Z chromosome - Dosage compensation . Sex determination $\cdot$ MHM valley
Abbreviations
MHM male hypermethylated region
DMRT1 doublesex and Mab-3-related transcription factor 1
RT- reverse transcriptase polymerase chain
PCR reaction
QPCR quantitative RT-PCR.

\section{The role of the avian $\mathrm{Z}$ and $\mathrm{W}$ chromosomes in sex determination is unclear}

In birds, the sex chromosomes are designated $\mathrm{Z}$ and $\mathrm{W}$, and the females possess a heterologous pair of sex chromosomes (ZW) while male sex chromosomes are homologous (ZZ). The chicken $\mathrm{Z}$ chromosome is approximately $74 \mathrm{Mb}$ long and well represented (with 840 genes identified) in the most recent (May 2006) build of the chicken genome sequence (International Chicken Genome Sequencing consortium 2004, Wahlberg et al. 2007), while the small and highly heterochromatic $\mathrm{W}$ chromosome is poorly represented with only $260 \mathrm{~Kb}$ of sequence available and only 4 genes identified. Despite the shared ancestry between 
the $\mathrm{Z}$ and $\mathrm{W}$ chromosomes, a lack of meiotic pairing along most of the length of the female-specific W chromosome has resulted in its degeneration in both size and sequence content. Lack of recombination between heterologous sex chromosomes is a general phenomenon in the evolution of differentiated sex chromosomes from autosomes, and is thought to be initiated by the isolation of a sex determining gene or genes on one proto-sex chromosome. The identity of the avian sex-determining gene remains unknown and there are two likely mechanisms for sex-determination in birds. A W chromosome based sex-determining mechanism would require a female-specific ovary determining gene located on the $\mathrm{W}$ chromosome, while a $\mathrm{Z}$ chromosome dosage-based system would be dependent on the number of $\mathrm{Z}$ chromosomes present ( $1 \times Z=$ ovary, $2 \times Z=$ testis). A number of potential avian sex determining genes have been identified and the currently most popular scheme proposes a dosage mechanism of sex determination (reviewed in Ferguson-Smith 2007), which depends on the conserved $\mathrm{Z}$ chromosome location of the DMRT1 gene in birds (Nanda et al. 2008). Interestingly, the $\mathrm{Z}$ and $\mathrm{W}$ chromosomes of ratite birds are almost homologous showing minimal $\mathrm{W}$ degeneration (Shetty et al. 1999) and are believed to represent an early stage of sex chromosome differentiation. The presence of DMRT1 on the ratite Z chromosome and its absence from the near homomorphic W chromosome (Shetty et al. 2002) is taken as strong evidence of the sex-determining credentials of this gene.

\section{The DMRT1 gene is a provocative but unconfirmed candidate for the key avian sex-determining gene}

The vertebrate DMRT1 (doublesex and Mab-3-related transcription factor 1) gene is thought to represent an evolutionary conserved sexual regulator owing to a shared ancestry with fly and worm sexual regulators (Raymond et al. 1998). Moreover, DMRT1 has a widespread and common role in sexual differentiation across vertebrate species, often showing temporally appropriate sexually dimorphic expression during differentiation of the male gonads (Raymond et al. 1999). Chicken DMRT1 is located on the $Z$ chromosome and is expressed at higher levels in male (ZZ) gonads than in female (ZW) gonads, both at and after the suspected point of sex determination on embryonic day 5.5 (Smith et al. 1999). Significantly, DMRT1 expression is elevated in embryonic female gonads 'masculinised' by the action of the aromatase inhibitor, fadrozole (Smith et al. 2003). However, despite the conserved role of DMRT1 as a downstream sexual regulator in male sexual development in many species, no precedent exists for DMRT1 as the key sexdetermining gene and the theory of DMRT1 as an avian sex-determining gene hinges on mapping of this gene specifically to the avian Z. Recent mapping data for other ZW species show that DMRT1 double dose can not represent a common mechanism of ZW sexdetermination since DMRT1 maps to both $\mathrm{Z}$ and $\mathrm{W}$ in some lizards (Kawai et al. 2009) and is autosomal in snakes (Matsubara et al. 2006) and ZW turtles (Kawai et al. 2007). Interestingly, the unusual multi-copy sex chromosomes (five XX or XY pairs) of the platypus show some conserved synteny with avian sex chromosomes (Grutzner et al. 2004; Rens et al. 2007). Here the DMRT1 gene is found on the $5^{\text {th }}$ platypus $\mathrm{X}$ chromosome (X5) (Veyrunes et al. 2008), and is present as a double-dose in female platypus and a single dose in males, which contrasts with the situation in birds. Perhaps sex-differential expression of this gene is important for sex determination in these disparate systems but, if so, the primary determinant cannot be double DMRT1 dosage in all cases.

One modification of the DMRT1 sex determination theory in birds assigns a role to the $\mathrm{Z}$ located noncoding RNA known as MHM (male hypermethylated region), which is only expressed in females (Teranishi et al. 2001). This RNA of unknown function, has been shown to accumulate at the site of transcription which is cytogenetically adjacent to the DMRT1 locus (Teranishi et al. 2001). It has been suggested that MHM RNA represses DMRT1 expression in females as part of the sex determination process (FergusonSmith 2007). However, to date there is no evidence of any direct interaction between MHM and DMRT1, nor evidence that MHM has any capacity to silence gene expression. Moreover, sequence information from the most recent build of the chicken genome (May 2006) predicts more than $1 \mathrm{Mb}$ of sequence and 11 other genes to lie between the two loci (Fig. 1).

The alternative hypothesis to a dosage-based mechanism of sex-determination holds that the $\mathrm{W}$ chromosome may carry an ovary-determining gene. The current paucity of sequence information available 


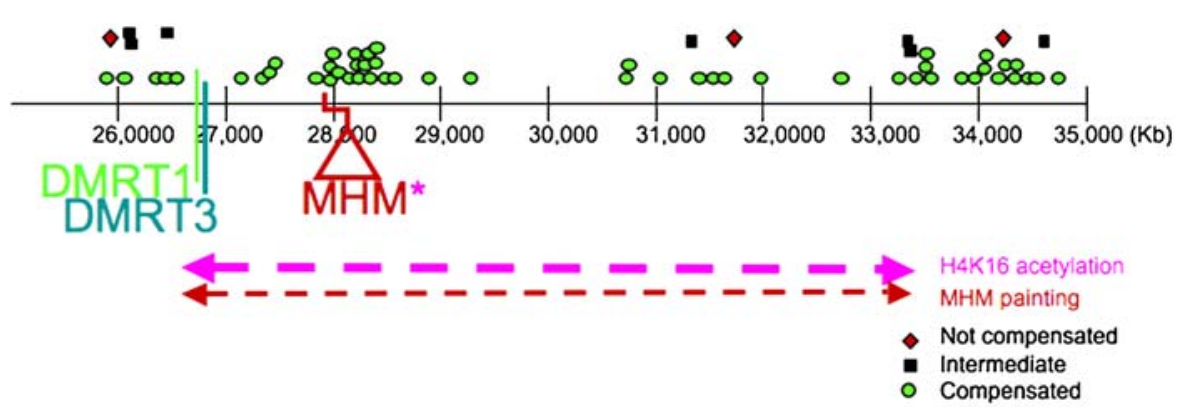

Fig. 1 The "MHM valley" region on female chicken $\mathrm{Zp}$ showing the positions and dosage compensation status of 61 genes. Genes positions are plotted along the map in kilobases from the $\mathrm{Zp}$ telomere, and symbols are attributed according to male to female ratios provided (Itoh et al 2007 supplementary data). Green circles denote dosage compensated genes with ratios of 1.3 or less in any tissue, red diamonds indicate noncompensated genes with ratios of 1.5 or above in all tissues tested and genes intermediate between these two categories are shown as black squares. Expression data was unavailable for DMRT1, DMRT3, and a further 33 annotated genes in this region which are not shown. Locations of the DMRT genes and the MHM gene are taken from the 2.1 build of the chicken genome (accessed 27/11/2008). The red triangle indicates the

for the $\mathrm{W}$ chromosome confounds full exploration of this possibility and the strongest $\mathrm{W}$ candidate to date (the HINTW gene reviewed in Smith 2007) has now been shown to lack dominant sex-determining potential during mis-expression experiments (Smith et al. 2009).

A further possibility exists that avian sex determination relies not on sex specific expression differences at a single locus but rather on a different pattern of expression at multiple loci between the two sexes, resulting from the different $\mathrm{ZZ}$ versus $\mathrm{ZW}$ sex chromosome constitutions.

\section{Female avian $\mathrm{Z}$ chromosome genes have a reduced gene dosage relative to autosomal and male $Z$ genes}

The evolution of heteromorphic ZW sex chromosomes in birds has the consequence that all female $\mathrm{Z}$ chromosome genes are monosomic. One would expect that such significant aneuploidy would not be easily tolerated (Birchler 2009) and recent studies in mammals and insects provide concrete evidence of the need to balance the expression of hemizygous sex chromosome genes with the expression of autosomes (Cheng and Disteche 2006). Genome-wide microarray expression analysis has recently demonstrated that male mammals achieve global upregulation of expression from their single $\mathrm{X}$ chromosome in order to equal the absence from this sequence of approximately $450 \mathrm{~Kb}$ of repetitive MHM sequence. The end of a $9 \mathrm{~Kb}$ region showing MHM homology $(27,963 \mathrm{~kb}$ to $27,972 \mathrm{~kb})$ coincides precisely with an annotated sequencing gap which is presumably approximately $450 \mathrm{~Kb}$ long to accommodate the missing MHM sequence. A further 19 gaps exist in the region shown. Pink and red broken lines along the bottom of the diagram indicate that this region is strongly acetylated at H4K16, and painted with non-coding MHM transcripts, specifically on the female Z. Neither the length nor the boundaries of these modified areas are known due to their cytogenetic detection. MHM* indicates that the MHM gene has been confirmed to be affected by the H4K16 acetylation while DMRT1 is unaffected

level of expression from autosomal genes, which are present in twice the dose (Nguyen and Disteche 2006a). Similarly for the nematode worm, despite the previously accepted dosage compensation mechanism of "double-down" regulation of expression from the two hermaphrodite $\mathrm{X}$ chromosomes, the single male $\mathrm{X}$ chromosome is now shown to be up-regulated to give a male $\mathrm{X}$ to autosome expression ratio of 1 (Gupta et al. 2006). In addition to, or perhaps as a result of, this sex chromosome to autosome balancing, further modifications occur to reduce sex chromosome expression in homogametic individuals, resulting in equalisation of expression between the sexes. Such modifications in mammals and insects are well studied (reviewed elsewhere in this issue) and the term "dosage compensation" is used to describe the normalisation of sex chromosome gene expression that results. The question then arises of whether birds also perform some form of dosage compensation in order to correct for the reduced gene dosage along the single female $\mathrm{Z}$ chromosome.

\section{Sex chromosome dosage compensation occurs in birds}

Until relatively recently dosage compensation was widely accepted to be absent in birds, principally 
on the basis of limited evidence regarding sexdifferential isozyme activity of the Z-linked aconitase protein (Baverstock et al. 1982). However, around 2001 this view was reversed when two smallscale studies established that the levels of expression of some $\mathrm{Z}$ chromosome genes were equalised in male and female birds, suggesting some form of dosage compensation (McQueen et al. 2001; Kuroiwa et al. 2002). Using the limited gene sequences and map locations available at that time, we examined transcript levels for a selection of nine genes dispersed along the chicken $\mathrm{Z}$ chromosome. Expression levels for all nine genes were analysed by quantitative RT-PCR (QPCR) in early (day 3 and day 4) male and female chick embryos (McQueen et al. 2001). Despite observing very heterogeneous levels of expression between the 12 or more individual male and female birds analysed for each gene, similar averaged levels of expression were found in males and in females for six of these genes (McQueen et al. 2001, Table 1). We concluded that these genes must be subject to some form of dosage compensation but that this regulation did not affect all $\mathrm{Z}$ chromosome genes, since at least one of the nine genes was clearly not compensated. Dosage compensation was also independently demonstrated for one of two additional genes studied in 15 day old embryos by a similar technique (Kuroiwa et al. 2002, Table 1). These studies established for the first time that a significant proportion of avian $\mathrm{Z}$ chromosome genes were subject to dosage compensation, but gave no real indication as to the extent of this compensation or to the nature of the mechanism involved. Such QPCR studies were advantageous in their ability to detect and present accurate levels of gene expression for individual genes in individual animals and to assess the variability that exists. The small number of genes that were measured could, however, lead to a skewed impression of the extent of dosage compensation. Moreover, while male: female ratios for $\mathrm{Z}$ gene expression were measured, the small number of control autosomal genes analysed precluded assessment of average $\mathrm{Z}$ chromosome: autosome $(\mathrm{Z}: \mathrm{A})$ ratios. While comparing expression levels of $Z$ chromosome genes in male and female tissues is useful in terms of monitoring the effects of dosage compensation, the expected role of dosage compensation would not be to achieve parity in $\mathrm{Z}$ gene expression between the sexes, but rather to regulate the sex chromosome to autosome ratio within individuals, similar to the recently demonstrated upregulation of the single male $\mathrm{X}$ chromosome to autosomal levels in mammals and nematode worms. The most informative measurement of the extent of sex chromosome dosage compensation in birds should, therefore, be the global sex chromosome to autosome expression ratio in the heterogametic sex, the female Z:A expression ratio.

\section{Average $\mathrm{Z}$ :A gene expression ratios are $>0.5$ but $<1$ in female birds indicating that dosage compensation is not chromosome-wide}

Results are now available from a number of medium- and large-scale studies of gene expression in male and female avian tissues giving $\mathrm{Z}: \mathrm{A}$ expression ratios which provide a more global picture of the extent of dosage compensation in birds. In zebra finch, a medium-scale analysis was performed where $40 \mathrm{Z}$ chromosome genes and 84 autosomal genes were analysed in 4 different adult and newborn tissues by competitive hybridisation with mixtures of male and female cDNA (Itoh et al. 2007). Although the experimental design of this study precluded analysis of $\mathrm{Z}$ : A ratios within the separate sexes, some degree of male over-expression was detected for $36 \mathrm{Z}$ located genes which argues against a chromosome wide system of tight dosage compensation. A larger scale analysis of chicken gene expression by the same authors used affymetrix chicken genome microarrays containing more than 16,000 probes of which around $5 \%$ represented $\mathrm{Z}$ chromosome genes. Gene expression was measured in brain, liver and heart from 20 male and 20 female day 14 embryos, with five sets of pooled samples from each sex being hybridised separately. Male: female expression ratios were established for individual genes, and used to generate an average male: female ratio of 1.2-1.4 for the Z-chromosome, while the average male:female ratio for autosomal genes was close to 1.0. In contrast, mammals were reported to show male:female expression ratios close to 1.0 for both autosomal and X-linked genes. Since the category of $\mathrm{Z}$ located genes showing male biased expression was shown to include housekeeping genes, the authors concluded that their results did 
Table 1 Dosage compensation status of 10 chicken $Z$ genes showing male to female ratios derived from 3 independent studies

\begin{tabular}{|c|c|c|c|}
\hline \multirow[t]{2}{*}{ Gene } & \multicolumn{2}{|c|}{ Position on the chromosome } & \multirow[t]{2}{*}{$\mathrm{m}: \mathrm{f}$ ratio } \\
\hline & cytogenetic & sequence $(\mathrm{kb})$ & \\
\hline Zov3 & $(\mathrm{p} 2.1)$ & $14,147-14,166$ & $1.35^{1}$ \\
\hline Follistatin & $(\mathrm{p} 2.2-2.3)$ & $15,391-15,398$ & $1.35^{1}$ \\
\hline $\operatorname{Brm}($ Smarca2) & $(\mathrm{p} 1.2)$ & $27,123-27,225$ & $1.23^{1}, 1.1^{3}, 1.3^{4}, 1.2^{5}$ \\
\hline VLDL & $(\mathrm{p} 1.2-1.3)$ & $27,352-27,367$ & $0.78^{1}, 1.0^{3}, 0.9^{4}, 0.6^{5}$ \\
\hline CHDZ & & $50,156-50,203$ & $1.23^{2}$ \\
\hline ChrnB/ACHB3 & $(q 1.3)$ & $52,500-52,510$ & $1.33^{1}$ \\
\hline AldoB & $(q 1.5)$ & $63,699-63,708$ & $0.94^{1}, 0.9^{3}, 0.9^{4}, 1.0^{5}$ \\
\hline ScII & $(q 1.5-1.6)$ & $65,031-65,032$ & $2.24^{1}$ \\
\hline Ggtb2/B4galt1 & $(q 1.5-1.6)$ & $68,712-68,722$ & $1.39^{1}, 2.09^{2}, 1.9^{3}, 1.9^{4}, 1.7^{5}$ \\
\hline \multirow[t]{2}{*}{ Irebp } & $(q 1.6)$ & $69,043-69,081$ & $0.83^{1}, 1.1^{3}, 1.1^{4}, 1.0^{5}$ \\
\hline & & Average: & $1.27^{1}$ \\
\hline
\end{tabular}

${ }^{1}$ day 3 and day 4 whole embryos analysed by QPCR (McQueen et al. 2001), ${ }^{2}$ day 15 embryo fibroblasts analysed by QPCR (Kuroiwa et al. 2002), ${ }^{3}$ day 14 embryonic brain analysed by microarray analysis (Itoh et al. 2007), ${ }^{4}$ day 14 embryonic heart analysed by microarray analysis (Itoh et al. 2007), ${ }^{5}$ day 14 embryonic liver analysed by microarray analysis (Itoh et al. 2007)

not simply reflect a preferential $\mathrm{Z}$ location for sexbiased genes, but instead provides evidence of "ineffective" dosage compensation of avian sex chromosomes (Itoh et al. 2007). However, the authors noted that the chicken Z:A ratios, which were consistently higher in males $(0.92-1.08)$ than females $(0.70-0.87)$, were nevertheless within the range of X:A ratios reported for mammals which does indeed suggest balancing of $\mathrm{Z}$ and $\mathrm{A}$ gene expression in birds (Itoh et al. 2007).

In a separate study, chicken affymetrix arrays were hybridised with day 18 embryonic heart, gonad and brain samples from each of 4 male and 4 female birds. Sexually dimorphic expression was reported for just under a quarter of all genes, of which $25 \%$ were located on the $Z$ chromosome (Ellegren et al. 2007). Male:female expression ratios were calculated for individual genes and the mean fold-change of $\mathrm{Z}$ gene expression in male versus female somatic tissues was reported as 1.42 (although a subsequent study by the same authors reported a reduced average male: female expression ratio of 1.24 in embryonic and adult brain (Mank and Ellegren 2009a)). Autosomal genes displayed no such sex-specific differences with a mean fold-change close to 1.0 (Ellegren et al. 2007). In contrast to the results of Itoh, Ellegren and colleagues described significantly elevated female A:
$\mathrm{Z}$ ratios, (corresponding to low Z:A ratios) quoting a $39 \%$ higher level of gene expression for autosomal versus $\mathrm{Z}$ chromosome genes in somatic tissue. This appears to be at odds with data presented in additional file 7 (Ellegren et al. 2007) which seems to show hybridisation intensities for the female $\mathrm{Z}$ chromosome that are in the same range as those from autosomes. The authors also noted that the overall average level of expression for dosage compensated $\mathrm{Z}$ chromosome genes tends to be lower than average levels of autosomal gene expression and conclude that this reflects down-regulation of male expression rather than up-regulation of female expression (Ellegren et al. 2007). This is a surprising finding given our assumption that the $\mathrm{Z}$ : A ratio would principally be a problem for the heterogametic females where upregulation of expression from the single $\mathrm{Z}$ chromosome might be expected.

It is clear from both studies that a significant number of genes across the avian $\mathrm{Z}$ are expressed at a higher level (although not normally 2-fold) from the two male $\mathrm{Z}$ chromosomes than from the single female $Z$ chromosome (Itoh et al. 2007; Ellegren et al. 2007). This strongly argues against global all-inclusive dosage compensation across the avian $\mathrm{Z}$ and suggests that many genes are not compensated, or only partially compensated. Importantly, both papers pres- 
ent graphs representing the spread of male: female expression ratios which have an obvious bimodal appearance rather than a continuous spread from low to high values. This implies that distinct populations of genes exist with respect to sex-specific gene expression on the $\mathrm{Z}$ chromosome. Presumably these discrete gene categories represent dosage compensated and non-compensated genes. Because of the separate and significant populations of compensated and non-compensated genes on the $\mathrm{Z}$ chromosome, the derived Z:A ratios each represent an average of disparate quantities which detracts from the value of such ratios. It is worthy of note, however, that the female Z:A expression ratio (reaching 0.87, (Itoh et al. 2007)) is significantly raised from the actual ratio of $\mathrm{Z}$ :A chromosomes $(0.5)$, in line with the conclusion that internal $\mathrm{Z}$ :A balance at many loci is maintained.

\section{Approximately $45 \%$ of $Z$ chromosome genes are dosage compensated}

Given that the male: female $\mathrm{Z}$ gene expression ratios from all the available studies range from less than 1 to more than 2, the classification of genes into compensated and non-compensated categories necessitates adoption of arbitrary cut-off values. Using the criteria stated in Melamed and Arnold 2007, (a ratio of $<1.3$ for a compensated gene and $>1.5$ for a noncompensated gene), a close examination of the male: female ratios of $\mathrm{Z}$ gene expression in the brain (presented in Melamed and Arnold 2007) allows us to estimate that approximately $45 \%$ of genes are dosage compensated, about $38 \%$ are clearly not dosage compensated and approximately $17 \%$ of genes show intermediate compensation in brain. In loose agreement with these values, Figure 2 in Itoh et al. 2007 shows only $20-50 \%$ of genes on the chicken $\mathrm{Z}$ to be more highly expressed in males than in females, while in Ellegren et al. 2007 the authors indicate that, for an imposed fold-change cut-off of 1.5, 25\% of $623 \mathrm{Z}$ chromosome genes analysed showed no male- biased expression in any tissue. Various interpretations of these studies have given rise to a bewildering array of apparently inconsistent conclusions which might, to some extent, have arisen through the use of different cut-off points and different use of terminology, but must also be affected by different experimental design.
When considering the evidence relating to dosage compensation, it is important to be able to distinguish inherent sexually dimorphic expression (relating to a lack of dosage compensation), from acquired sexually dimorphic expression (relating to sex-specific functional differences). Because of male-specific or female-specific functions, many tissues display sexually dimorphic expression unrelated to the issue of dosage compensation. There is no obvious reason why this sexually dimorphic expression cannot apply as readily to $\mathrm{Z}$ chromosome genes as to autosomal genes and such tissues should be avoided in dosage compensation analysis. The most obvious example to avoid is the adult gonads: while, in general terms, the ovary and testis perform the same function in males and females (the nurture and delivery of gametes), in morphological, biochemical and molecular terms, these are two distinctly different organs. Even in terms of the relative proportions of germ cells and somatic tissues, the ovary and testis are markedly different and any sexually dimorphic expression is more likely to be related to functional differences than to the issue of dosage compensation. This is evident in the scatter-plot comparison of autosomal gene expression in adult male and female gonads (Ellegren et al. 2007). Adult brain and liver are two other tissues known to have sex-specific functional differences and concomitant sex-differential expression in both chickens and mammals (Scholz et al. 2006; Nguyen and Disteche 2006b). To avoid sexuallydimorphic expression resulting from secondary sexspecific functional differences, we suggest that the most appropriate material for dosage compensation analysis is whole embryo at a stage prior to any sexual differentiation (for example, chick embryos between day 2 and day 4 of development).

Another variable that must be considered is that the majority of chicken lines studied are not inbred and, as noted in our original analysis (McQueen et al. 2001), the expression levels of individual genes varies considerably, even between individuals of the same sex. Consequently the most reliable average expression values will be generated by the studies that include the greatest number of individual birds, such as the analysis of 20 birds from each sex (Itoh et al. 2007).

While microarray analyses are necessary to generate the required large-scale datasets, these techniques are widely accepted to be less accurate than more 
labour-intensive QPCR analyses making confirmation by PCR highly desirable. Despite the small number of genes sampled, results from QPCR experiments (McQueen et al. 2001; Kuroiwa et al. 2002, Table 1), are in agreement with the conclusion that different populations of genes exist (with respect to dosage compensation). Five of the 10 genes originally analysed meet our new criteria for dosage compensation, and the average male: female ratio for all 10 genes is 1.27 , in good agreement with the larger datasets. There are microarray generated male: female ratios available for five of the 10 genes originally measured by QPCR (Table 1), and reassuringly, all five genes give similar values by both techniques (Table 1).

From all the studies available, it now seems reasonable to conclude that, despite the apparent absence of chromosome wide dosage compensation, some form of dosage compensation is at work in birds, affecting approximately $45-60 \%$ of $\mathrm{Z}$ chromosome genes.

\section{Dosage compensated genes are distributed across the chromosome with a significant cluster on chicken $\mathbf{Z p}$}

In describing this partial and frequently incomplete pattern of dosage compensation, both Itoh and Ellegren question whether the mechanisms involved need to be sex chromosome specific, and propose that intrinsic genome wide regulatory systems may be sufficient to correct for the imbalance in expression. However, such whole chromosome aneuploidy corrected only by intrinsic compensation normally results in lethality or a highly abnormal phenotype (Birchler 2009). A non sex-chromosome-specific compensation system would dispense with the need for local sex chromosome mechanisms of compensation and eliminate the possibility of regions of the $\mathrm{Z}$ chromosome sharing local compensation profiles. However, Melamed and Arnold (2007) have demonstrated that strongly compensated and non-compensated regions do occur, and highlight a specific cluster of compensated genes in the proximal region of the short arm of $\mathrm{Z}$ (Fig. 1). This finding is disputed by Mank and Ellegren 2009a, on the basis of a further microarray expression analysis of chicken brain and gonad. This study relies on further statistical analyses to propose an "ad hoc" system of dosage compensation, where the compensated state varies depending on tissue type and stage of development, but states that no neighbourhoods of overall dosage compensation could be detected (Mank and Ellegren 2009a). Using our previously stated criteria for compensated and noncompensated genes, 51 of the 61 genes in the $\mathrm{Zp}$ region analysed by Melamed and colleagues do indeed show dosage compensation in at least one tissue, while only 3 genes lack compensation in all tissues (Melamed and Arnold 2007, supplementary data) (Fig. 1). This is a particularly interesting region of the $\mathrm{Z}$ chromosome as it contains both the DMRT1 gene and the MHM non-coding RNA gene. Had the MHM gene been included in this expression data-set, its female specific expression might have skewed the results towards an overall lower male: female ratio (as suggested by Mank and Elegren (2009a)). However, less than $2 \%$ of the repetitive MHM gene sequence is present in the 2.1 build of the chicken genome sequence (May 2006) (Fig. 1) and this portion of the MHM gene is absent from the Melamed data-set. Instead, the low male: female expression ratio of this region reflects a genuine predominance of tightly dosage compensated genes (Fig. 1, Melamed and Arnold 2007). Such clustering of compensated genes in this so-called "MHM valley" is strongly supportive of the concept of active regional compensation on the $\mathrm{Z}$ chromosome rather than genome wide regulatory influences. Importantly, only two of the five randomly located dosage compensated genes analysed in the original QPCR experiments (Table 1) lie within the "MHM valley", indicating that dosage compensation also occurs frequently outwith this cluster on $\mathrm{Zp}$.

\section{The dosage compensation mechanism in birds is likely to involve chromatin modifications but not $\mathrm{Z}$ inactivation}

Although there is disagreement as to the extent of dosage compensation, all the studies performed to date have presented evidence of avian dosage compensation, which raises the question as to the nature of the mechanism employed in birds. If we look to the best-studied model systems (mammals, Drosophila, and C. elegans), we can see large scale or global X chromosome dosage compensation being achieved by a variety of different mechanisms including $\mathrm{X}$ 
chromosome up-regulation, $\mathrm{X}$ chromosome inactivation and $\mathrm{X}$ chromosome down-regulation (reviewed in this issue). Since these modifications must be maintained at cell division, it is not surprising that stably inherited epigenetic chromatin modifications, such as histone acetylation and/ or methylation changes, are frequently employed. Interestingly, upregulation of the single male $\mathrm{X}$ in Drosophila, and random inactivation of one of the two female mammalian X chromosomes both involve not only histone modifications but additional chromatin associated non-coding RNAs (rox and Xist respectively). Rox and Xist are entirely distinct in sequence and are components of two very different systems. In both cases however, the sex specific RNA is specifically transcribed from, and paints, the target sex chromosome and both RNAs are considered necessary for appropriate targeting of the dosage compensation machinery (reviewed in Akhtar 2003). Marsupial and monotreme mammals are also known to execute dosage compensation (also reviewed in this issue) but, interestingly, the patterns of compensation differ from those of their eutherian counterparts. Specifically, marsupial female somatic cells display an incomplete and unstable version of $\mathrm{X}$ inactivation, which always inactivates the paternally derived copy (Wakefield et al. 1997). Partial and variable dosage compensation, akin to that described for birds, has recently been demonstrated for genes located on the chain of monotreme $\mathrm{X}$ chromosomes where female: male expression ratios range from 1.0 to 2.0 and the overall average ratio is approximately 1.3 (Warren et al. 2008, supplementary Figure 5). Transcription analyses by RNA-FISH, for a small number of platypus $\mathrm{X}$ chromosome genes, demonstrates monoallelic transcription in a portion of cells, leading to a mixture of monoallelically and biallelically expressed cells and intermediate transcript levels (Deakin et al. 2008). This monoallelic expression is shown to be co-ordinated on a regional level for two genes separated by $500 \mathrm{~Kb}$ (Deakin et al. 2008). The Xist gene is absent from all non-eutherian sex chromosomes.

In birds, we know that any mechanisms of dosage compensation are unlikely to include inactivation of one $\mathrm{Z}$ chromosome since it has long been known that no avian sex chromatin (inactive sex chromosome) is visible and that the two male $\mathrm{Z}$ chromosomes replicate synchronously (Schmid et al. 1989). Moreover, biallelic expression has been demonstrated by nascent RNA-FISH experiments for five Z-linked genes (Kuroda et al. 2001) including the aconitase and aldolaseB genes which have been confirmed as tightly dosage compensated in all tissues studied (McQueen et al. 2001; Itoh et al. 2007, Table 1). Although gene numbers are small, the lack of cells expressing a single allele (as seen in the platypus) argues for wholesale biallelic expression of avian $\mathrm{Z}$ genes, and against Z-inactivation.

\section{Strong dosage compensation coincides with an activating chromatin modification and accumulation of non-coding RNA, both specific to the female avian $Z$}

To date, no sex chromosome specific chromatin changes have been demonstrated to be unequivocally associated with avian dosage compensation. However, a female Z-specific chromatin modification and accumulation of a female Z-specific non-coding RNA have been observed and seem particularly worthy of note considering the relevance of similar features to dosage compensation systems in other species. Firstly, a series of antibody staining experiments on embryonic and adult chicken cells demonstrated striking female-specific staining of the $\mathrm{Z}$ chromosome with an antibody specific to Histone H4 modified by acetylation of the $16^{\text {th }}$ residue (lysine) (Bisoni et al. 2005). This antibody staining is particularly exciting to note since the same chromatin modification is specifically enriched on the up-regulated male Drosophila $\mathrm{X}$ chromosome, where it is known to be associated with hypertranscription. All current theories of male X upregulation for dosage compensation in Drosophila depend on the presence and specificity of this modification. In contrast to the chromosome-wide location of this modification in Drosophila, in birds the modification is strongly staining only on one localised portion of the short arm of the female $\mathrm{Z}$ chromosome which coincides with the "MHM valley", noted for its high density of dosage compensated genes. One hypothesis would be that this female sex chromosome specific chromatin modification in birds is necessary to upregulate gene expression within the "MHM valley" in order to effect the tight dosage compensation observed in this region. Perhaps the low resolution of the antibody staining technique is insufficient to detect the modification of 
compensated genes elsewhere on the female $\mathrm{Z}$ which are not so tightly clustered, but which could be detected by further chromatin immunoprecipitation experiments. Alternatively, perhaps the "MHM valley" represents a sub-compartment of the $\mathrm{Z}$ chromosome which is regulated separately from other more dispersed genes undergoing dosage compensation by different means. A precedent for the co-existence of discrete dosage compensation systems exists in drosophila (Fitzsimons et al. 1999).

The second piece of circumstantial evidence which may be relevant to effecting dosage compensation pertains to the MHM gene itself. Also giving a female-specific pattern of expression, and specifically methylated and silenced on the male $\mathrm{Z}$ chromosome (Teranishi et al. 2001), the MHM region has been frequently suggested to have an important role in avian sex determination as discussed above. However, no evidence exists to support interaction of MHM with the DMRT1 gene and the function and mode of action of MHM remains to be elucidated. The wide and varied functions of the newly emerging class of large non-coding RNAs (reviewed in Umlauf et al. 2008) gives us no clue as to the purpose of local MHM accumulation on the $\mathrm{Z}$ chromosome. By analogy with the sex chromosome located Xist and rox non-coding RNA genes, one possibility could be targeting of local chromatin with chromatin modifying complex for the purpose of dosage compensation. In addition to the MHM non-coding transcript painting the local $\mathrm{Z}$ chromatin (Teranishi et al. 2001), chromatin immunoprecipitation experiments show that the MHM gene (but not the nearby DMRT1 gene) is itself affected by the localised H4K16 acetylation modification (Bisoni et al. 2005). Taken together with the knowledge that this area represents a high density region of fully dosage compensated genes (Melamed and Arnold 2007), this accumulated evidence starts to suggest a model whereby the MHM RNA has a role in targeting genes for dosage compensation by female specific hypertranscription. It would not be surprising to find a noncoding RNA in such a role, given the well studied Xist and rox precedents, both with a role in targeting completely different dosage compensation machineries to the sex chromosome, yet both associated with entirely distinct outcomes (Kelley and Kuroda 2000).

\section{Incomplete dosage compensation in birds makes DMRT1 one of many differentially expressed genes}

The issue of sex-chromosome dosage compensation is crucial to our understanding of sex determination in birds. Implicit in the DMRT1 gene dosage theory for sex determination is that expression of the DMRT1 double gene dose is uncorrected by dosage compensation and that this state is exceptional such that outwith the sex-determining locus, the levels of expression of $\mathrm{Z}$ chromosome genes are equalised in males and females. If all $\mathrm{Z}$ chromosome genes are expressed at twice the level in males (ZZ) as in females $(Z W)$, then the significance of higher levels of DMRT1 expression in males is diminished.

The location of the DMRT1 gene within the "MHM valley" is worthy of note. No DMRT1 expression was detected by QPCR in the day 3 and 4 embryonic studies (McQueen et al. 2001) and male: female ratios are not available for the DMRT1 gene from any other dosage compensation studies. To remain uncorrected by dosage compensation, DMRT1 would need to be protected from any putative locally operating factors in the "MHM valley". Lack of H4K16 acetylation of DMRT1 chromatin, despite strong H4K16 acetylation of the surrounding region, has indeed been demonstrated (Bisoni et al. 2005). The large numbers of chicken genes not corrected by dosage compensation sits well with the idea that DMRT1 gene dosage may remain non-compensated, but reduces the significance of this gene dosage difference since a large number of other $\mathrm{Z}$ chromosome genes appear to show some level of sex-differential expression. Such significant and widespread sex-specific variation in $\mathrm{Z}$ chromosome expression might lead us to re-consider a model of sex determination which is not dependent on the differential expression of a single sex chromosome gene but of multiple $\mathrm{Z}$ chromosome genes.

\section{Conclusion}

Despite many years of study, we still lack significant understanding of the processes of sex determination and dosage compensation in birds. Our knowledge of sex chromosome dosage compensation relies on a limited number of studies and many of the conclusions drawn to date are, at best, tentative and often speculative. The current data suggests a picture 
whereby a proportion of the $\mathrm{Z}$ chromosome genes are subjected to some form of dosage compensation but that this is not a sex chromosome-wide phenomenon. In terms of relative male:female expression levels, $\mathrm{Z}$ chromosome genes seem to fall into three broad categories; i) genes that are expressed at similar levels in males and females (compensated), ii) genes that are expressed at approximately 2-fold higher levels in males than in females (not-compensated), and iii) genes with an intermediate male:female expression ratio ("ineffectively" compensated). From the available data, it appears that the proportion of genes in each category is approximately $45 \%, 38 \%$ and $17 \%$ respectively and the two main categories may be reflected in the bimodal appearance of the graphed expression data published by Itoh and by Ellegren. Theoretically, genes in category (i) would be detrimental if expressed at different levels in males and females, genes in category (ii) perhaps require to be expressed at higher levels in males (as would a dosage dependent sex-determining gene), while genes in category (iii) result in no detrimental effects if expressed at different levels in males and females.

Of significant note is the "MHM valley" on the short arm of the $\mathrm{Z}$ chromosome that shows consistently strong dosage compensation and co-incident epigenetic modifications, hinting at a regional dosage compensation mechanism to specifically increase gene expression from the single female Z. Compensated genes outwith this region could be similarly modified or, alternatively, two or more separate mechanisms could be at work to modify expression at different loci across this sex chromosome. It is interesting to note that the region of clustered tight dosage compensation on the chicken $\mathrm{Z}$ chromosome does not coincide with the most ancient stratum which lies across Zq, while the "MHM valley" resides in the area of $\mathrm{Z}$ described as "the major avian radiation" (Handley et al. 2004). Perhaps the highly acetylated and strongly dosage compensated "MHM valley" represents not an ancient conserved mechanism but a newly emerging system, yet to spread across the chromosome, and specific to a subset of avian species. Indeed, the presence of MHM has only been tested in Galliformes to date (Teranishi et al. 2001). What is certain is that this is an exciting time for the avian sex chromosomes with many questions still to be answered, not least the function and relevance of this peculiar MHM region.
Acknowledgements HMcQ is funded by the Wellcome Trust, and MC by BBSRC and DEFRA.

\section{References}

Akhtar A (2003) Dosage compensation: an intertwined world of RNA and chromatin remodelling. Curr Opin Genet Dev 13:161-169

Baverstock PR, Adams M, Polkinghorne RW, Gelder M (1982) A sex-linked enzyme in birds-Z-chromosome conservation but no dosage compensation. Nature 296:763-766

Birchler JA (2009) Dosage compensation for the birds. Heredity Feb 18. [Epub ahead of print]

Bisoni L, Batlle-Morera L, Bird AP, Suzuki M, McQueen HA (2005) Female-specific hyperacetylation of histone $\mathrm{H} 4$ in the chicken Z chromosome. Chromosome Res 13:205-214

Cheng MK, Disteche CM (2006) A balancing act between the $\mathrm{X}$ chromosome and the autosomes. J Biol 5:2

Deakin JE, Hore TA, Koina E, Marshall Graves JA (2008) The status of dosage compensation in the multiple $\mathrm{X}$ chromosomes of the platypus. PLoS Genet 4:e1000140

Ellegren H, Hultin-Rosenberg L, Brunstrom B, Dencker L, Kultima K, Scholz B (2007) Faced with inequality: chicken do not have a general dosage compensation of sex-linked genes. BMC Biol 5:40

Ferguson-Smith M (2007) The evolution of sex chromosomes and sex determination in vertebrates and the key role of DMRT1. Sex Dev 1:2-11

Fitzsimons HL, Henry RA, Scott MJ (1999) Development of an insulated reporter system to search for cis-acting DNA sequences required for dosage compensation in Drosophila. Genetica 105:215-226

Grutzner F, Rens W, Tsend-Ayush E et al (2004) In the platypus a meiotic chain of ten sex chromosomes shares genes with the bird Z and mammal X chromosomes. Nature 432:913-917

Gupta V, Parisi M, Sturgill D et al (2006) Global analysis of Xchromosome dosage compensation. J Biol 5:3

Handley LJ, Ceplitis H, Ellegren H (2004) Evolutionary strata on the chicken $\mathrm{Z}$ chromosome: implications for sex chromosome evolution. Genetics 167:367-376

International Chicken Genome Sequencing Consortium (2004) Sequence and comparative analysis of the chicken genome provide unique perspectives on vertebrate evolution. Nature 432:695-716

Itoh Y, Melamed E, Yang X et al (2007) Dosage compensation is less effective in birds than in mammals. J Biol 6:2

Kawai A, Nishida-Umehara C, Ishijima J, Tsuda Y, Ota H, Matsuda Y (2007) Different origins of bird and reptile sex chromosomes inferred from comparative mapping of chicken Z-linked genes. Cytogenet Genome Res 117:92-102

Kawai A, Ishijima J, Nishida C et al (2009) The ZW sex chromosomes of Gekko hokouensis (Gekkonidae, Squamata) represent highly conserved homology with those of avian species. Chromosoma 118:43-51

Kelley RL, Kuroda MI (2000) Noncoding RNA genes in dosage compensation and imprinting. Cell 103:9-12

Kuroda Y, Arai N, Arita M et al (2001) Absence of Zchromosome inactivation for five genes in male chickens. Chromosome Res 9:457-468 
Kuroiwa A, Yokomine T, Sasaki H et al (2002) Biallelic expression of Z-linked genes in male chickens. Cytogenet Genome Res 99:310-314

Mank JE, Ellegren H (2009a) All dosage compensation is local: gene-by-gene regulation of sex-biased expression on the chicken Z chromosome. Heredity 102:312-320

Mank JE, Ellegren, H (2009b) Sex-linkage of sexually antagonistic genes is predicted by Female, but not male, effects in birds. Evolution, Jan 14. [Epub ahead of print]

Matsubara K, Tarui H, Toriba M et al (2006) Evidence for different origin of sex chromosomes in snakes, birds, and mammals and step-wise differentiation of snake sex chromosomes. Proc Natl Acad Sci U.S A 103:18190-18195

McQueen HA, McBride D, Miele G, Bird AP, Clinton M (2001) Dosage compensation in birds. Curr Biol 11:253-257

Melamed E, Arnold AP (2007) Regional differences in dosage compensation on the chicken $\mathrm{Z}$ chromosome. Genome Biol 8:R202

Nanda I, Schlegelmilch K, Haaf T, Schartl M, Schmid M (2008) Synteny conservation of the $\mathrm{Z}$ chromosome in 14 avian species ( 11 families) supports a role for $Z$ dosage in avian sex determination. Cytogenet Genome Res 122:150-156

Nguyen DK, Disteche CM (2006a) Dosage compensation of the active $\mathrm{X}$ chromosome in mammals. Nat Genet 38:4753

Nguyen DK, Disteche CM (2006b) High expression of the mammalian X chromosome in brain. Brain Res 1126:4649

Raymond CS, Shamu CE, Shen MM et al (1998) Evidence for evolutionary conservation of sex-determining genes. Nature 391:691-695

Raymond CS, Kettlewell JR, Hirsch B, Bardwell VJ, Zarkower D (1999) Expression of Dmrtl in the genital ridge of mouse and chicken embryos suggests a role in vertebrate sexual development. Dev Biol 215:208-220

Rens W, O'Brien PC, Grutzner F et al (2007) The multiple sex chromosomes of platypus and echidna are not completely identical and several share homology with the avian $\mathrm{Z}$. Genome Biol 8:R243

Schmid M, Enderle E, Schindler D, Schempp W (1989) Chromosome banding and DNA replication patterns in bird karyotypes. Cytogenet Cell Genet 52:139-146
Scholz B, Kultima K, Mattsson A et al (2006) Sex-dependent gene expression in early brain development of chicken embryos. BMC Neurosci 7:12

Shetty S, Griffin DK, Graves JA (1999) Comparative painting reveals strong chromosome homology over 80 million years of bird evolution. Chromosome Res 7:289-295

Shetty S, Kirby P, Zarkower D, Graves JA (2002) DMRT1 in a ratite bird: evidence for a role in sex determination and discovery of a putative regulatory element. Cytogenet Genome Res 99:245-251

Smith CA, McClive PJ, Western PS, Reed KJ, Sinclair AH (1999) Conservation of a sex-determining gene. Nature 402:601-602

Smith CA, Katz M, Sinclair AH (2003) DMRT1 is upregulated in the gonads during female-to-male sex reversal in $\mathrm{ZW}$ chicken embryos. Biol Reprod 68:560-570

Smith CA (2007) Sex determination in birds: HINTs from the W sex chromosome? Sex Dev 1:279-285

Smith CA, Roeszler KN, Sinclair AH (2009) Genetic evidence against a role for W-linked histidine triad nucleotide binding protein (HINTW) in avian sex determination. Int J Dev Biol 53:59-67

Teranishi M, Shimada Y, Hori T et al (2001) Transcripts of the MHM region on the chicken $\mathrm{Z}$ chromosome accumulate as non-coding RNA in the nucleus of female cells adjacent to the DMRT1 locus. Chromosome Res 9:147-165

Umlauf D, Fraser P, Nagano T (2008) The role of long noncoding RNAs in chromatin structure and gene regulation: variations on a theme. Biol Chem 389:323-331

Veyrunes F, Waters PD, Miethke P et al (2008) Bird-like sex chromosomes of platypus imply recent origin of mammal sex chromosomes. Genome Res 18:965-973

Wahlberg P, Stromstedt L, Tordoir X et al (2007) A high-resolution linkage map for the $\mathrm{Z}$ chromosome in chicken reveals hot spots for recombination. Cytogenet Genome Res 117:22-29

Wakefield MJ, Keohane AM, Turner BM, Graves JA (1997) Histone underacetylation is an ancient component of mammalian X chromosome inactivation. Proc Natl Acad Sci U S A 94:9665-9668

Warren WC, Hillier LW, Marshall Graves JA et al (2008) Genome analysis of the platypus reveals unique signatures of evolution. Nature 453:175-183 